\title{
Estimation of long-term stability of detectors of the world neutron monitor network for the whole monitoring period
}

Belov Anatoly

Pushkov Institute of Terrestrial Magnetism, Ionosphere and Radio Wave Propagation, Russian Academy of Sciences, IZMIRAN, Moscow, Russia, 108840; E-mail: abeloveizmiran.ru

\section{Dorman Lev ${ }^{1}$}

Israel Cosmic Ray and Space Weather Center Institute of Advanced Study of Tel Aviv University, P.O. Box, 39040, Tel Aviv, ISRAEL, 69978; IZMIRAN; E-mail: 1id0105290gmail.com

\section{Gushchina Raisa}

Pushkov Institute of Terrestrial Magnetism, Ionosphere and Radio Wave Propagation, Russian Academy of Sciences, IZMIRAN, Moscow, Russia, 108840; E-mail: rquseizmiran.ru

\section{Eroshenko Eugeniya}

Pushkov Institute of Terrestrial Magnetism, Ionosphere and Radio Wave Propagation, Russian Academy of Sciences, IZMIRAN, Moscow, Russia, 108840; E-mail: erosheizmiran.ru

\section{Preobrazhensky Maxim}

Pushkov Institute of Terrestrial Magnetism, Ionosphere and Radio Wave Propagation, Russian Academy of Sciences, IZMIRAN, Moscow, Russia, 108840; E-mail: planeswalker.ipsumegmail.com

\section{Yanke Victor}

Pushkov Institute of Terrestrial Magnetism, Ionosphere and Radio Wave Propagation, Russian Academy of Sciences, IZMIRAN, Moscow, Russia, 108840; E-mail: yankeeizmiran.ru

\begin{abstract}
Worldwide neutron monitor network today consists of more than 50 neutron monitors. To provide long time observation of solar activity revealed in cosmic rays the first importance has a stable operating of the neutron monitor network. The method of this long time stability estimation is considered in presented work. The quantitative ratios of stable operation and causes of the long time drifts in the neutron monitor data are presented for each station. Now days the new scientific and applying problem stand before the network, so, this network is essentially modernized at present. The main task now is to provide the network operating and operating as a single multidirectional device with sufficiently stable long time accuracy.
\end{abstract}

35th International Cosmic Ray Conference - ICRC2017

10-20 July, 2017

Bexco, Busan, Korea

1

\section{Speaker}




\section{Introduction}

More than half a century, researchers of cosmic ray variations have used such a powerful instrument as the global network of stations equipped with neutron monitors. The aim of the present work is to determine the quality of data obtained at each station. Such a problem was also stated previously [Belov et al., 2005; Belov et al., 2007]. To estimate quantitatively the quality of instrumental data, it is necessary to have a standard. Researchers often use a groundless approach using any reliably operating station as such a standard.
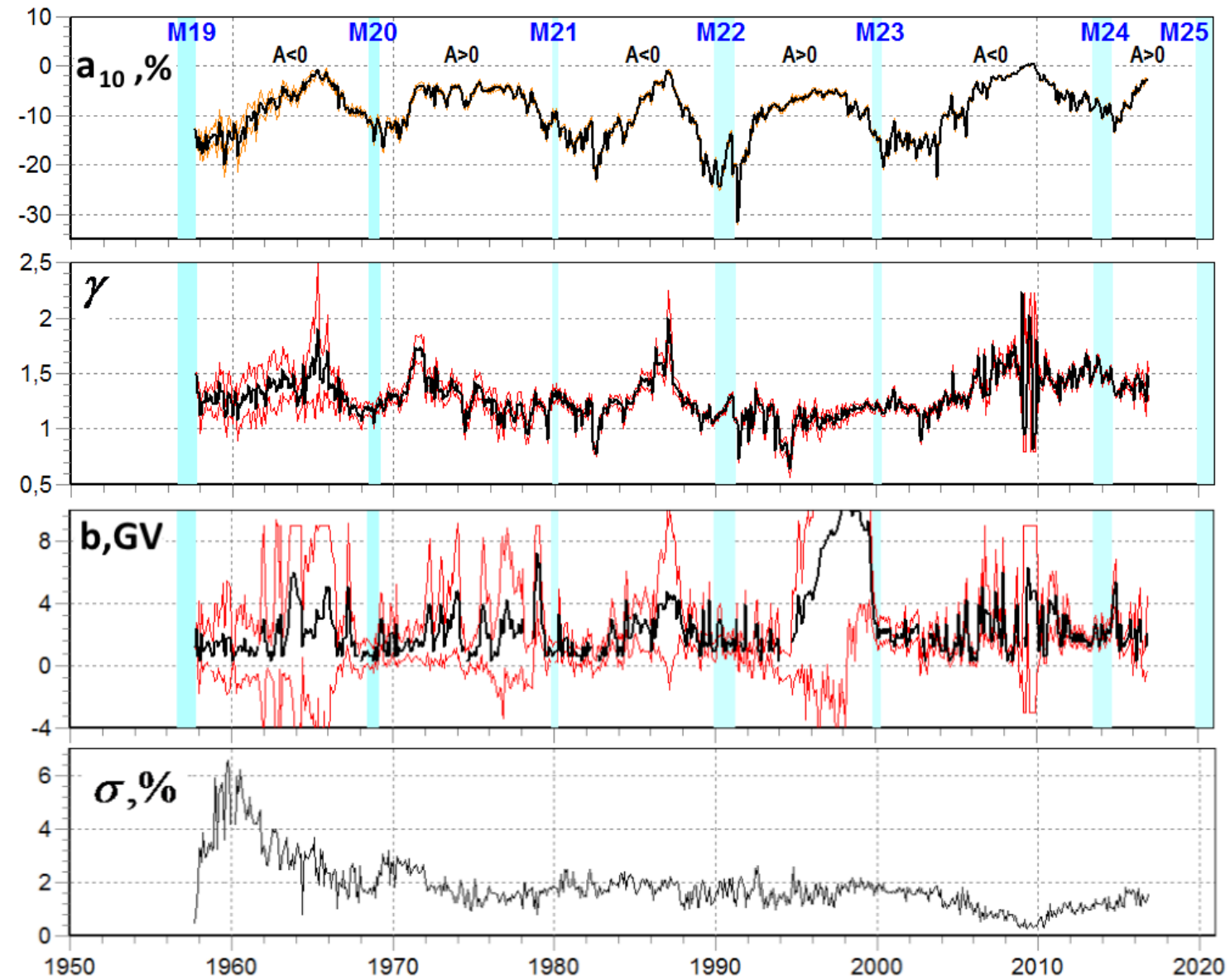

Figure 1. Parameters of the galactic cosmic rays variation spectrum: $a_{10}, \gamma, b$ and $\sigma$ the model discrepancy is shown at the bottom.

Indeed, many stations have been reliably operating but cannot be used as a standard since each station registers cosmic rays in a specific spectral region. In this paper we used two independent methods. For the first method the model of cosmic ray variation developed as a standard. A difference from the model for each station is related to the quality of data for this station. Certainly, such an approach has its own disadvantages since it is difficult to construct an adequate model. However, this problem can be solved by using the method of consecutive approximations if it is possible to describe sufficiently adequately cosmic ray variations in the heliosphere for the 60-year period of observations with the help of appropriate model modifications. Another independent method for estimating station operation stability, which can be called the method of ratios [Belov et al., 1988], was developed in addition to the model method. With the help of the method of ratios and the developed mathematical algorithm, a specified set of stations (with approximately identical effective rigidities of registered particles, 
$\mathrm{R}_{\text {eff }}$ ) is divided into the groups of reliably and unreliably operating stations. In such a case a

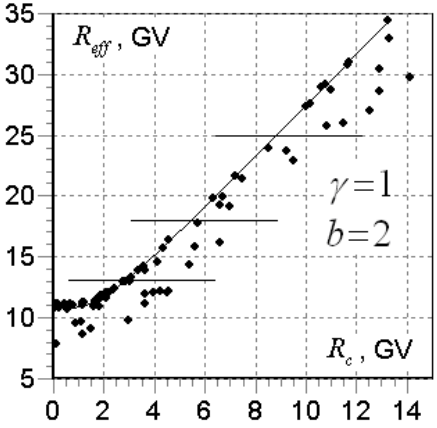

Figure 2. Effective particle rigidity for different stations; the sealevel and mountain stations are shown on the curve and below the curve, respectively. group of reliably operating stations with identical variations up to statistics (and the number of such stations should be predominant) is used as a standard. For each station, the method of ratios makes it possible to determine effectiveness and, which is of prime importance, the accuracy of effectiveness determination. However, the effectiveness of a detector can sometimes be determined only using a model approach. For example, to use the method of ratios, it is necessary to have more than three identical stations. Therefore, the effectiveness of three or two operating stratospheric stations, as well as that of sparse equatorial neutron monitors, can be determined only based on a model approach. Either method has its own advantages and limitations; however, a joint usage of both methods makes it possible to obtain reliable quantitative estimates of a long-term stability of neutron monitors within the global network of stations. An analysis was performed based on the average monthly data obtained by averaging the hourly data from the database of the global network of neutron monitors [Data Base, 1997]. The aim of the work is to estimate a long-term stability of operation of the neutron monitors within the global network of cosmic ray stations by using two independent methods.

\section{Model approach}

In paper [Belov et al., 1998] determination the spectrum of long-term cosmic ray variations by using the version of the globally spectrographic method, which was specially modernized in order to study long-term variations. An analysis was performed based on the
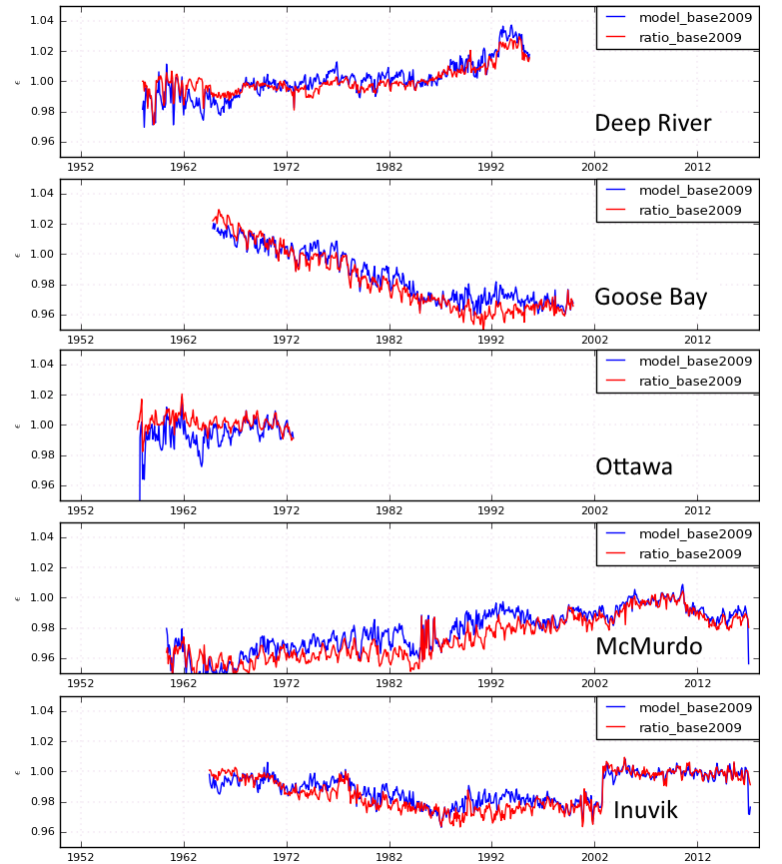

Figure 3 Effectiveness obtained using the model method (blue) and the method of ratios (red). The level of 2009 was taken as a unit. average monthly data of neutron monitors (45 stations), muon telescope, stratospheric observations (three stations). An observed variation $\delta I^{i} / I^{i}$ can be represented as $\frac{\delta I^{i}}{I^{i}}=$ $\int_{R_{c}}^{\infty} W\left(R, R_{c}, h^{i}\right) \cdot \frac{\delta J}{J}(R) d R^{+} \sigma^{i}$, where $\delta J J(R)$ is the spectrum of isotropic variations, and the discrepancy $\sigma^{i}$, reflects inadequacy of the used model of variations and possible instrument variations. The coupling functions $W^{i}\left(R, R_{c}, h^{i}\right)$ are calculated by us and for the neutron component taken from [Aleksanyan et al., 1982] and for the muon component taken from [Fujimoto et al., 1976]; here, $R_{c}$ is the geomagnetic threshold, and $h^{i}$ is the altitude. The model spectrum of variations was specified in the parametric form and determined as 
$\delta J / J(R)=a_{10} /(b+R)^{\gamma}$. The range of variations in the parameters is $\gamma=0 \div 2$ and $b=(0 \div 4) \mathrm{GV}$. The above work indicated that a three-parameter approximation of the galactic cosmic ray variation spectrum can be used to describe the spectrum of long-term variations within 5-50 GV. Only several stations continuously operated during five solar cycles. Some stations stopped their operation, and new stations were commissioned. The spectrum of variations was determined for one base period of 2009, which allowed avoiding an additional error that occurs when data is stitched when using different databases. Figure 1 shows the obtained parameters of the galactic cosmic ray variation spectrum $a_{10}, \gamma$ and $b$. The lower panel presents the standard deviations of the experimental and model data that make it possible to estimate adequacy of the applied model of cosmic ray variations.

\section{Method of ratios}

This method was initially developed in order to perform an internal quality control of ground-based cosmic ray detectors. A two-sectional nm57 monitor (where one could only qualitatively evaluate the instrumental variations) was replaced by a three-sectional nm64

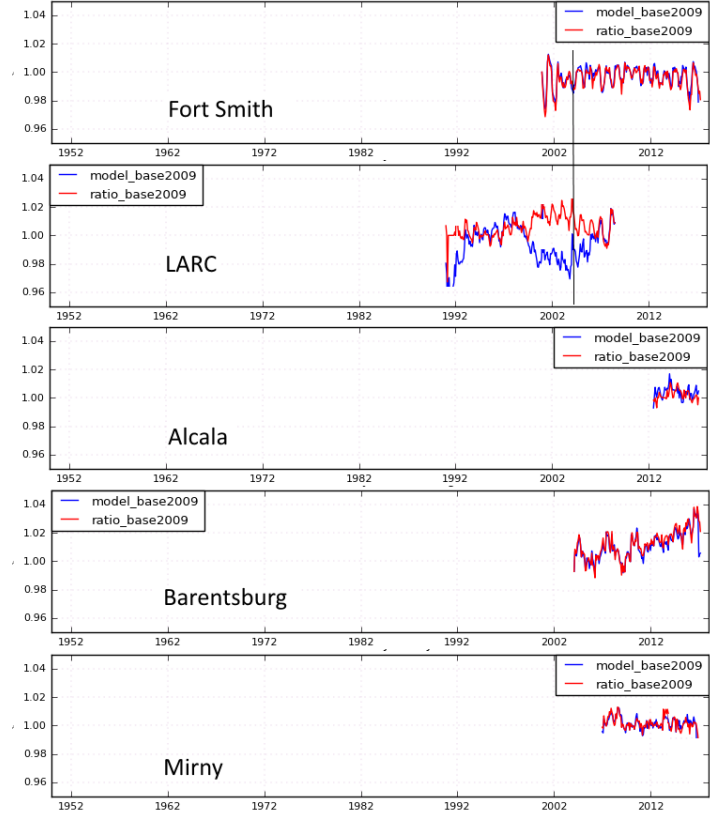

Figure 4 Effectiveness obtained using the model method (blue) and the method of ratios (red). The level of 2009 was taken as a unit. neutron monitor, for which a comparison of variations at different sections of the same type makes it possible to distinguish a defective section. Up-to-date methods of internal control are based on division of a detector into the maximum possible number of identical and independent elementary detectors (neutron counters in the case of a neutron monitor). Such an approach makes it possible to determine the relative effectiveness (and the effectiveness error) for each elementary detector [Belov et al., 1988]. The detector effectiveness can be determined as a number into which the observed count rate should be divided in order to get rid of variations related to changes in the detector itself. The method of ratios was modified so that it could be used to analyze the long-term stability of detectors at the network of stations. The method of ratios can be applied if stations with very close characteristics (e.g., with close effective rigidities of registered particles) are combined in one group. We considered four groups of stations with effective rigidities of particles $<13,13-18,18-25$, and $>25 \mathrm{GV}$ (Figure 2). The fact that this approach does not use any model is its advantage. However, the necessity of considering groups of stations with close effective rigidities of registered particles is the method disadvantage. To take into account the time variations in geomagnetic cutoff rigidities, we made calculations performed for the network of cosmic ray stations [Gvozdevsky et al., 2016]. 


\section{Discussion of results}

Figure 3 shows the variations in the effectiveness for certain stations, obtained using the model method and the method of ratios. For all cosmic ray stations, such information is presented on the site [Animation; LongTimeVariation, 2017]. A coincidence in details of effectiveness found by two methods argues for both methods. If there is a difference, this requires analysis: either the model for these energies does not work well, or the method was not in its group. An analysis of effectiveness indicates that a constant drift of $\pm 0.1 \%$ per year is observed at many stations

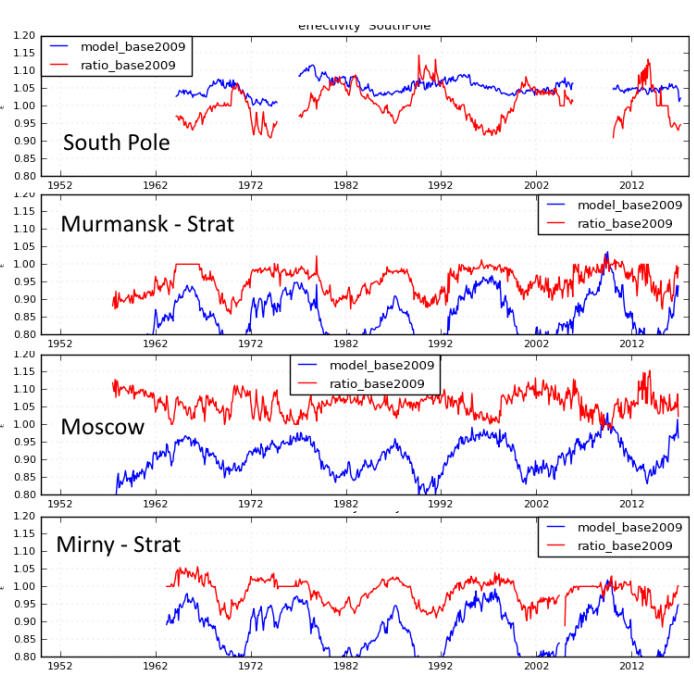

Figure 5 Effectiveness obtained using the model method (blue) and the method of relations (red). The level of 2009 was taken as a unit. during the entire or a rather long period of observations (e.g., at Deep River station, Fig. 3). The maximal drift (about $-0.4 \%$ per year) was detected for Goose Bay station. The annual wave with an amplitude of about $1 \%$, opposite in phase in the Northern and Southern hemispheres (Ottawa and McMurdo stations, in archive [LongTimeVariation, 2017]), is clearly defined at high-latitude stations. This wave is caused by the temperature effect of the neutron component [Belov et al., 1995] and is negligible for equatorial stations. An anomalously large annual temperature effect $(2-4 \%)$ is observed at a number of stations (e.g., Beijing, Tibet station) (it presented on the site [LongTimeVariation, 2017]). Part of this effect (less than 1\%) is related to the temperature effect of the neutron component; the remaining part, to a local change in the temperature affecting the electron tract elements. A typical change in the effectiveness is shown in Fig. 3, which evidently demonstrates how the effectiveness varied when an nm57 detector was replaced by an nm64 one (1964). Variations in the effectiveness are mainly sporadic, apparently, due to the human factor.

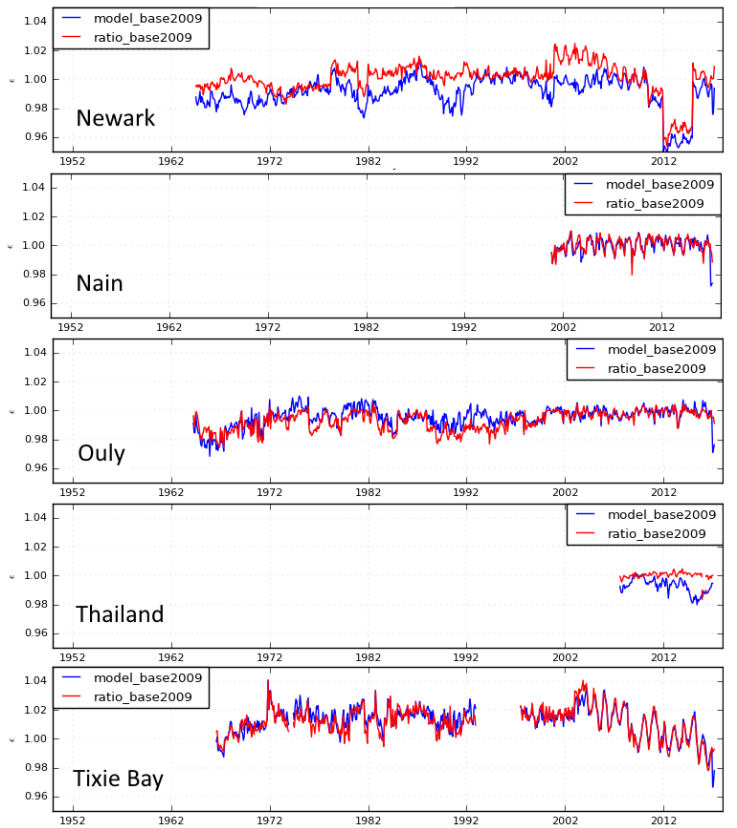

Figure 6 Effectiveness obtained using the model method (blue) and the method of relations (red). The level of 2009 was taken as a unit. Instrument variations (or drifts) can be classified as:

(1) diurnal and seasonal variations related to temperature changes,

(2) longterm variations related to a change in detector properties, and

(3) sporadic instrument drift.

If periodic variations and longterm instrumental drift can be easily distinguished, then it is extremely difficult to make sporadic instrumental drift.

The maximal error in data originates due to a drift in pressure (up to $0.1 \%$ per year). The usage of a constant barometric coefficient results in a false 11year wave with an amplitude larger than $0.1 \%$. Considerable sporadic changes in effectiveness can be caused by at least two factors: (1) charge leakage (micro breakdowns) in a high-voltage circuit and (2) insufficient stability of high-voltage power sources (or malfunction of 
regulating circuits). Moreover, the effect of snow is of prime importance for some mountain stations. This effect can result in a complete distortion of variations.

In Fig. 4 the efficiency of newly commissioned neutron monitors is shown. For highlatitude stations, an annual wave with an amplitude of about $1 \%$ is clearly observed, which is opposite in phase in the northern and southern hemispheres (Fig. 4: Fort Smith and LARC vertical line; in archive [LongTimeVariation, 2017] we can see Ottawa and McMurdo). It is due to the temperature effect of the neutron component [Belov et al., 1995] and is negligible for equatorial stations.

In Fig. 5 the effectiveness of stratospheric detectors with the South Pole neutron monitor is depicted. The ratios method works unstably because of the small number of such stations. The model method also works poorly in this energy range. In Fig. 6 the efficiency of some other detectors is plotted.

\section{Conclusions}

Based on the aforesaid, we can make the following conclusions:

(1) A model approach, when disagreement of cosmic ray variations with a model for each station is related to the station data quality, makes it possible to qualitatively and quantitatively estimate the long-term stability of operation of each cosmic ray station within the network. The accuracy depends on the adequacy of the used model of cosmic ray variations.

(2) The method of ratios is independent of a model. In this case the long-term drift is determined for each station in the group of stations with close energies. However, not all simultaneously operating identical stations can compose a sufficiently large (more than six stations) group, which is the disadvantage of such an approach.

(3) A coincidence in details between effectiveness found by two methods of ratios indicates that both methods are applicable. The model of cosmic ray variations adequately describes cosmic ray modulation during the entire 60 -year period of observations, maybe, except the region of low rigidities. This can be exemplified by South Pole station or stratospheric measurements.

(4) For stably operating stations (10-15), the drift can reach $0.05 \%$ / year. We compare it with the change in the amplitude of the variations (Fig. 1), obtained with the use of all network data for the entire observation period; the change is $\sim 1 \%$, i.e. $\sim 0.01 \%$ / year.

(5) Only about 25 stations have been operating for several tens of years with a stability of less than $2 \%$ during the entire period of observations. However, sporadic variations in effectiveness are typical for many of these stations, for which data drift is of a secondary significance and data quality depends on numerous sporadic variations. A typical drift of effectiveness is about $\pm 0.1 \%$ per year.

(6) For many stations (about 30), the drift of data is generally in the background, the quality of the data is determined by numerous sporadic changes.

(7) The operation of the network of cosmic ray stations is completely analyzed in [LongTimeVariation, 2017].

\section{Acknowledgments}

This work was partially supported by the Program of the Presidium of RAS № 23 "High energy physics and neutrino astrophysics", RFBR grant № 17-02-00508, experimentally and methodologically support the project UNU №85 "Russian national network of ground stations of cosmic rays" We are grateful to all the staff of the World Network of cosmic ray stations: http://nmdb/eu and http://cr0.izmiran.ru/ThankYou.

\section{References}

Asipenka A., Belov A., Gushchina R., Eroshenko E., Kobelev P., Yanke V., "Estimation of detectors stability of the neutron monitors network", Proc. of the 31st ICRC, Lodz, 2009. $\triangleq \underline{\mathrm{pdf}}$ | 
Aleksanyan T.M., Belov A.V., Yanke V.G., et al., "Experimental studies of geomagnetic effects in cosmic rays and the spectrum of the effect of increasing before magnetic storms". Izv. RAS. Ser. phys., V. 46, №9, p.1689-1691, 1982.

Data Base of neutron monitors network idb, ePub, 1997. http://cr0.izmiran.ru/common/links.htm

Belov A.V., Bloch Y.L., Klepach E.G., Yanke V.G., In book "Kosmicheskie luchi”, Moskow, №25. P.113, 1988; See also ftp://crsb.izmiran.ru/Data_Editor.

Belov A.V., Gushchina R.T., Eroshenko E.A., Yanke V.G., Geomagnetism and Aeronomy, V.38, №4, P.131, 1998.

Belov A., Gushchina R., Eroshenko E., Yanke V., "Estimate of long-term detector stability of the worldwide neutron monitor network", Proc. of the 29th ICRC, Pune, V.2, 239-242, $2005 . \mid$ $\triangleq$ pdf .

Belov A.V., Dorman L.I., Gushchina R., Yanke V.G. "Temporal and latitudinal dependence of the temperature effect for neutron component of cosmic ray", Proc. of the 24th ICRC, Rome, V.4, P.1141-1144, 1995.

Belov A.V., Gushchina R.T., Eroshenko E.A., Yanke V.G., "Estimation of long-term stability of detectors within the global network of neutron monitors", Geomagnetism and Aeronomy, V.47, No 4, Issue 2, P.251-255, 2007. doi:10.1134/S0016793207020144 / ISSN 00167932. 스 pdf

Fujimoto K., Murakami k., Kondo I., Nagashima K., "Calculation of Response Function for Cosmic Ray Hard Component at Various Depths of the Atmospheric and Underground". Proc. of ICRS on High Energy Cosmic Ray Modulation. University of Tokyo, Japan, p.50, (1976); // "Approximate formula for response function of cosmic ray hard component at various depths of the atmospheres and underground", Proc 15-th ICRC, Plovdiv, Bulgaria., V.4, 321-325, (1977).

LongTimeVariation, ftp://crsb.izmiran.ru/LongTimeCRVariation , 2005.

LongTimeVariation, ftp://crsb.izmiran.ru/LongTimeCRVariation/2017/Animation_effectivity_CR_net.gif , 2017. 